\title{
Erika Prijatelj
}

University of Ljubljana, Slovenia

\section{Itinéraire d'un bouc emissaire}

\section{A Scapegoat's Journey}

\begin{abstract}
Sacrifice is an important part of religious, ethical and political life. In addition, the term;sacrifice' has several meanings; one of them denotes a victim of crime. In this paper, we focus exclusively on victims of scapegoating. Scapegoats - individuals or communities - may be victims of others, or otherwise they may experience themselves in this way. This paper explores both possibilities. It, primarily studies the psychology of scapegoating based on the premise of the innate human need to discharge tensions, and then presents several paths out of the scapegoating mechanism. It also illustrates scapegoating dynamics in the media and explores the example of Slovenia based on its history and the current situation with respect to transferring of blame.
\end{abstract}

\section{Keywords}

Scapegoat, victim, tension, personal image, relationship.

\section{Introduction}

Selon Theodore Zeldin « rien n'a autant paralysé l'intelligence humaine que la recherche de boucs émissaires». ${ }^{1}$ Ainsi, notre question de départ est la suivante: qu'est-ce qui peut bien se passer aujourd'hui chez les gens lorsque le mécanisme

1 T. Zeldin, An Intimate History of Humanity, New York 1995, Harper Perennial, p. 36. 
du bouc émissaire s'empare des individus ou des sociétés, quand il leur devient impossible de percevoir des progrès concrets dans la résolution de cette dynamique. Nous verrons aussi, comment cette dernière s'est manifestée dans certaines cultures anciennes.

\section{2- bouc émissaire, victime d'autrui}

À l'origine, un bouc émissaire était une véritable chèvre. Une fois l'an, le grand prêtre juif présidait un rituel pendant lequel les intervenants déposaient symboliquement sur une chèvre les péchés de la communauté, puis l'expulsaient dans le désert. De cette façon, espéraient-ils, la chèvre emporterait le mal et les gens recommenceraient leur vie à zéro. Le rituel réussirait si les fidèles étaient disposés à se repentir de leurs péchés et à effacer les offenses qu'ils avaient causées aux membres de la communauté. Ce faisant, ils croyaient que Dieu pardonnerait leurs fautes et les reconnaîtrait à nouveau comme son peuple. Malgré le fait que les Juifs sacrifiaient des animaux à leur Dieu, contrairement à d’autres communautés religieuses voisines, «la chèvre nétait pas liquidée, mais seulement chargée et expulsée». ${ }^{2}$

À cet égard, la chèvre «nétait pas une victime physique, mais porteuse du message» ${ }^{3}$ que Dieu avait ôté le fardeau de leurs péchés. Les Juifs, tout comme les autres communautés, voulaient restaurer leur sentiment d'appartenance, et partager l'expérience de soulagement, puisque le mal qui avait causé des divisions était expurgé. Il est significatif que, dans cette pratique, l’animal, (ou un être humain chez la plupart des autres religions de lère biblique), jouant le rôle du bouc émissaire, devait être innocent. Quand un humain devenait bouc émissaire, il s’agissait généralement d'un étranger ou d'un individu que la communauté percevait comme un demeuré ou comme l'un de ses membres le plus faible.

Le fait que le bouc émissaire des Juifs restait vivant est particulièrement important pour l'étude de nos pratiques contemporaines, puisque les victimes sont souvent rejetées de la communauté ou de la société, mais pas totalement détruites. La société a besoin de victimes, exploitées comme objets de récriminations; et,

${ }^{2}$ R. Raven, The Dorr War - Treason, Rebellion and the Fight for Reform in Rhode Island, Charleston 2010, Narrative Press, p. 92.

3 T. Douglas, Scapegoats - Transferring Blame, London 1995, Routledge, p. 29. 
parce qu'elles sont présentes, la société peut toujours continuer de les accuser d'un quelconque méfait tout en les ostracisant. Qu'ils soient des parias de leur famille, ou ceux de minorités ethniques, de communautés religieuses, ou qu'ils soient stratégiquement ciblés parce qu'ils ont "peur dêtre exposés à la vindicte publique». ${ }^{4}$ Compte tenu de la nature des médias modernes, qui sadonnent à traiter des affaires publiques, cette peur nest en rien irrationnelle. Les médias essaient de servir particulièrement l'intérêt général, vérifient la crédibilité des paroles et des actions des personnalités publiques et réagissent fortement lorsqu'il y a apparence d'irrégularité ou de fraude. En même temps, les médias sont vulnérables et souvent dépendants des autorités, qui représentent la vie sociale et politique.

Étant donné que les médias sont les instruments qui créent l’image publique des élites, ils n'en sont pas nécessairement des évaluateurs objectifs. Cela peut biaiser leur impartialité: certaines personnes sont transformées en super-héros, tandis que d'autres, sans motifs apparemment réels, le sont en parias. Avec l'aide des médias, le mécanisme du bouc émissaire dans la vie politique publique est rapidement déclenché lorsqu'une personne au pouvoir se sent menacée. Craignant dêtre dénoncée et d’avoir publiquement à justifier ses actions, elle peut également se faire aider par des médias aptes à détourner l'attention vers d'autres cibles, qui deviennent alors le nouveau centre d'intérêt. Ce qui rend impossible une confrontation avec le vrai mal et la véritable catharsis.

\section{3- bouc émissaire, perception de soi-même}

Tout au long de l'histoire humaine, la société a souvent adopté une attitude hargneuse à légard des victimes. Le christianisme a également joué un rôle dans le soutien de cette mentalité en occultant le malaise des personnes visées, en leur promettant une récompense après la mort. Dans ce contexte, il présentait le Christ essentiellement comme une icône tragique de la souffrance et, par conséquent, encourageait les victimes à souffrir et à soffrir en sacrifice d'oblation au Christ - sans, en même temps, aider à éliminer la cause première de leur souffrance. Aujourd'hui, la culture occidentale, qui, à bien des égards plus que dans le passé ou dans les sociétés non occidentales, a développé une sensibilité respectueuse et compatissante envers les victimes, fait face à d'autres

4 T. Douglas, Scapegoats - Transferring Blame, London 1995, Routledge, p. 47. 
défis. Certains de ces défis se présentent sous de nouvelles formes de victimes (qu'elles soient des cyber-victimes, des victimes identitaires, etc.) et leur offrent une assistance ajustée à leurs besoins. Néanmoins, l'un des principaux défis pour la société occidentale est de savoir comment reconnaître et aider véritablement les individus qui souffrent d’être des boucs émissaires, de soigner l'image de soi des victimes et de faire appel à une société «culturellement encline à favoriser des personnalités narcissiques $»^{5}$ bien que porteuses d'aide. Dans une société d'individus à la personnalité narcissique, lexpérience d'un bouc émissaire peut facilement aboutir à la conclusion «qu'en retour de son intervention, on se sent en droit d'avoir certains privilèges, ce qui renforce davantage les attitudes et les comportements égoïstes». ${ }^{6}$

Dans notre société postchrétienne, nous sommes confrontés au retour du sens tragique de la vie, associé au désespoir. Ce désespoir affiche des aspects différents tels que la dépendance à la drogue de masse, à l'Internet, au consumérisme, et au suicide en augmentation croissante, etc. Regarder la vie sociale à partir d'une perspective tragique crée un sentiment d'impuissance et déclenche lapitoiement sur son sort qui paralyse les victimes et les empêche de faire des efforts pour s'en sortir; ainsi, colère, frustration et approche négative de la vie s'accumulent. Zitek a prouvé scientifiquement ces résultats grâce à ses études sur de nombreux cas. Ainsi, des travailleurs ayant vécu des situations victimaires et perdu injustement des sommes d'argent, en raison d'une promotion d'emploi qui leur a échappé, exigent maintenant des salaires plus élevés que ceux qu'ils avaient dans le passé, alors qu'ils ne s'estimaient pas sous-payés. Le sens de léquité de leurs droits soi-disant bafoués, a produit, en retour, un comportement autocentré. Selon Zítek, ceux, qui ne connaissent pas la guérison intérieure et ne se remettent pas de leur malheur, trouvent toujours une excuse pour s'esquiver de certaines responsabilités sociales, ou, alors, sont moins enclins à aider leur entourage que ceux qui ne se sont pas vus comme bouc émissaire dans le passé. En somme, les personnes blessées mais non guéries expriment des besoins plus égocentriques et exigent une plus grande part de compensation que les autres ${ }^{7}$ (Zitek, 251-253).

${ }^{5}$ D. Kettle, Engaging with Tragic Spirituality and Victim Sensibility: on the Cultural Setting of Mission in the West Today, in: Mission Studies 21, p. 288.

${ }^{6}$ E. Zitek, Victim Entitlement to Behave Selfishly, "Journal of Personality and Social Psychology", February 2010, 98 (2), p. 247.

${ }^{7}$ E. Zitek, Victim Entitlement to Behave Selfishly, "Journal of Personality and Social Psychology", February 2010, 98 (2), p. 251-253. 


\section{4- psychologie de bouc émissaire}

"Le bouc émissaire a toujours eu le pouvoir mystérieux de libérer le plaisir féroce de l'homme de torturer, de corrompre et de nuire». ${ }^{8}$ Ce phénomène se produit surtout lorsqu'il s'agit de quelqu'un d'étranger à une communauté bien identifiée ou membre d'une communauté qui ne tolère pas la diversité en son sein. Storr, '9 qui a étudié le paradoxe de l'intolérance et du bouc émissaire chez les minorités, a constaté qu'elles deviennent des victimes parce quelles sont dans une position de faiblesse, alors quelles sont encore potentiellement puissantes et donc dangereuses. Storr soutient également que, en raison de leur capacité à s'identifier à une autre personne, ces gens sont, dans une certaine mesure, conscients de la souffrance des autres. Cependant, cette conscience même, qui accompagne l'identification avec l'autre, est source de compassion humaine mais aussi de cruauté.

Lorsqu'on choisit un "vrai " bouc émissaire, un mélange de sentiments, personnels ou collectifs, apparaît: frustration, colère, perception de la diversité, préjugé, soupçon, ignorance, peur, insécurité, projection, culpabilité, anxiété; ces sentiments sont d'autant plus pervers si on les laisse nous envahir et si on n’assume aucune responsabilité pour ses propres égarements. Ces différents sentiments, «en tant que source d'énergie, d'hostilité, d'envie et de différence sont extrêmement puissants» ${ }^{10}$. Dans une société frustrée, il n'en faut pas davantage pour déclencher une avalanche d'hostilité latente. En conséquence, certaines personnes deviennent rapidement des boucs émissaires sujets à des attaques verbales ou même à des sévices corporels.

Quand une source de frustration, ponctuelle ou continue, hors du contrôle individuel ou sociétal, se manifeste, il peut en résulter de la colère ou de l'hostilité, à la recherche d'un exutoire libérateur. Une impression qu'il n’y a pas de solution au problème soulève un degré élevé de tension, qui « exige » le passage à l'acte. Pour diverses raisons, les gens ne savent pas toujours comment se libérer, d'une façon ajustée, de tensions accablantes. L'alternative souvre sur la colère et la violence, attitude qui s'attaque particulièrement aux plus vulnérables. En outre, la violence comme réponse à la frustration est contagieuse.

${ }^{8}$ F. Mauriac, Child Martyrs (Second Thoughts), New York 1961, Publisher unknown, p. 14.

9 A. Storr, Human Aggression, New York 1968, Free Press, p. 98-99.

10 T. Douglas, Scapegoats - Transferring Blame, London 1995, Routledge, p. 119. 
Des études ${ }^{11}$ suggèrent que, dans une société où la réponse d'un individu à la frustration est agressive, le nombre augmente de ceux qui, dans des situations similaires, réagissent également avec violence.

Pour saisir le phénomène du bouc émissaire, il est, d'un point de vue psychologique, important de prendre en considération les "parties inacceptables de soi, et la projection de ces dernières sur son entourage, lequel peut se sentir incité à épouser ces traits, comportements et intentions inacceptables». ${ }^{12}$ Cela signifie que, lorsque l'on éprouve des difficultés à résister à ses pulsions négatives, c'est-à-dire en proie à une lutte pour accepter leurs aspects néfastes, cela provoque un sentiment inconfortable dont on cherche à se libérer. Le moyen le plus rapide pour libérer cette détresse est la persécution de ceux dans lesquels on voit les mêmes faiblesses. Et, alors, le mécanisme de bouc émissaire s'installe insidieusement. C’est le transfert sur l'autre de la responsabilité de ses propres tendances. Dans les situations qui traitent de la complexité de l'expérience humaine et de la division simpliste entre le bien et le mal, surtout en l'absence d'une réflexion appropriée et d'une incapacité à prendre en charge ses propres actions, le mécanisme du bouc émissaire peut se manifester tant au niveau d'un individu que des groupes, des organisations, et des communautés, religieuses ou non.

Nous pouvons conclure que lêtre humain a un „besoin inné” ${ }^{13}$ de libérer la tension qui se développe en raison de la perception de ses fonctionnements personnels ou sociaux inacceptables. Ce besoin, auquel les sociétés contemporaines sont confrontées maintenant, ressemble encore à celui des communautés d'autrefois. La différence en est que la société laïque occidentale d'aujourd'hui ne peut plus dire qu'elle craint le rejet ou la punition d'un Dieu vengeur, qu'elle ne prend plus au sérieux. Cependant, aujourd'hui, la peur du rejet et de la punition publique demeure encore souveraine. Les gens craignent que, directement ou indirectement, la société ou le système ne les punissent, surtout s'ils estiment qu'ils n'appartiennent pas à un segment social puissant ou privilégié ou parce qu'ils osent critiquer la société ou se distancier de ses modèles et de ses attentes.

\footnotetext{
11 J. Campbell, Reflections on the Art of Living, San Anselmo, CA 2011, Campbell, p. 117.

12 T. Douglas, Scapegoats - Transferring Blame, London 1995, Routledge, p. 117.

13 J. Campbell, Reflections on the Art of Living, San Anselmo, CA 2011, Campbell, p. 181.
} 


\section{5- bouc émissaire, bourreau d'autrui}

Avant de répondre à la question de savoir comment surmonter la prise en compte de la dynamique du bouc émissaire, nous examinerons ce qui se passe chez les gens créateurs des conditions d'activation du mécanisme du bouc émissaire.

En tant qu'individus, les gens créent sur eux-mêmes des récits qu'ils croient conformes à la réalité. Ce sont généralement des récits sécurisants qui agrémentent la notion d'image de soi. Ils façonnent leurs souvenirs en en gardant certains et en rejetant ceux qui ne leur conviennent pas. Cela se vérifie dans le phénomène que les psychologues qualifient de "penchant affirmatif». ${ }^{14}$ Les explications et les excuses sont créées rationnellement, bien que toujours sous l'influence de leurs émotions. En raison de l'importance qu'ils s'attribuent, ils se sentent spéciaux et extraordinaires; par conséquent, ils surestiment facilement leurs capacités dans divers aspects de la vie. Par exemple, un sondage a révélé que $80 \%$ des gens interrogés croient qu'ils sont de meilleurs conducteurs de voitures que d'autres. ${ }^{15}$ Néanmoins, il faut considérer qu'autant de gens ne peuvent pas être des conducteurs exceptionnellement bons, sinon il n'y aurait pratiquement aucun accident de la circulation. Mais, convaincus qu'ils sont de très bons conducteurs, ils croient qu'ils ne sont pas responsables de ce qu'un accident peut leur arriver, ni de ses conséquences.

Plus ils se trompent sur eux-mêmes, plus ils tentent de protéger l'image idéalisée qu'ils se sont forgée, tout en blâmant les autres pour leurs erreurs. Par conséquent, dans leur esprit, les mauvaises situations ne peuvent pas leur être imputées mais sont causées par d’autres personnes ou événements; par ailleurs, ils attribuent leur succès à leurs talents naturels.

La capacité de blâmer les autres se développe tôt, au début de la vie. L'enfant colérique qui tempête et frappe du pied envoie un message à l'adulte. S'il ne se fait jamais corriger, plus tard, en tant quadulte, il renforcera cette attitude par des excuses telles que: « Ce nétait pas moi. C’était la boisson ». Ou: « Je ne l’aurais jamais fait si on ne m’avait pas dérangé. » De même, on peut penser à combien meilleure serait l'existence d'une personne si, à la suite d'un décès d'un être cher, elle avait pu auparavant obtenir de lui un soutien positif; ou, si une personne,

14 J. Campbell, Reflections on the Art of Living, San Anselmo, CA 2011, Campbell, p. 181.

${ }^{15}$ M. J. Liersch, I am a Better Driver than You Think: Examining Self-Enhancement for Driving Ability, "Journal of Applied Social Psychology" (2014) 43 (8), p. 1658. 
aux allures d'un bouc émissaire, ne seétait pas immiscé négativement, dans sa vie. Dans les deux cas, un bouc émissaire peut prendre racine.

Pour pouvoir vivre sa vie de façon responsable moralement, il est nécessaire d'assumer la responsabilité de ses propres paroles et actions, d'accepter la vie telle qu'elle est avec ses aléas, et d'aller au-delà de soi-même et de ses intérêts purement égoïstes. La psychologie de la nature humaine révèle la tendance naturelle à chercher un privilège pour soi-même, même si cela nuit à son développement humain. La tendance naturelle à se privilégier est enracinée dans les instincts primaires de l'auto-préservation et de l'estime exagérée de soi. Elle peut également être fondée sur «les échecs de l'imagination, les horizons étroits de l’attention qui sont parfois constitués par l'habitude et les mécanismes défensifs et trompeurs qui rendent aveugles les personnes», ${ }^{16}$ et les poussent à déconsidérer les motifs d'autrui de se comporter et, donc, de ne pas entrer en vraie relation avec lui.

La prise de conscience de nos propres zones d'ombre est cruciale pour aborder le mécanisme du bouc émissaire car, avec elle, il devient moins probable que nous les projetions sur l'autre. Lorsque nous sommes prisonniers de nos propres zones d’ombres, il est beaucoup plus difficile d'identifier le mécanisme qui se manifeste, dautant plus que nous en sommes moins conscients. Prisonniers dans nos propres zones dombres, les gens que nous avons subconsciemment choisis comme boucs émissaires peuvent facilement être caractérisés à un niveau conscient de «méchants». Les médias peuvent même contribuer à présenter une personne identifiée comme un monstre ou une sorcière et utiliser tous les détails pour la diaboliser davantage, la placer en position dégradante et lui donner peu de chances d'éviter les situations douloureuses.

Voici comment l'itinéraire du bouc émissaire pourrait être tracé: moins de récit imaginaire sur nous-mêmes ou sur nos communautés et plus d’approche de la réalité; moins de recherche des privilèges pour nous-mêmes et plus d'acceptation de notre responsabilité personnelle; plus de laisser-aller de notre supériorité (consciente et subconsciente) et moins de regard condescendant sur les autres (consciemment et subconsciemment).

${ }^{16}$ M. Halbertal, On Sacrifice, Princeton 2012, Princeton University Press, p. 64. 


\section{6- bouc émissaire de soi-même, sortie de la dynamique}

La persécution peut s'opérer soit sur autrui ou soit sur soi-même. Autant il est difficile de reconnaitre le mécanisme du bouc émissaire lorsqu'il est dirigé sur d'autres, autant il est difficile de le voir lorsqu'il est dirigé sur soi-même. Cela devient particulièrement difficile quand les deux opèrent simultanément. C'est le cas de la Slovénie qui, au cours de son histoire, a fait face à de nombreux problèmes économiques et politiques. La Slovénie a eu tendance à blâmer d'autres nations lors de situations précaires et, par conséquent, elle s’est perçue comme jouant le rôle d'un bouc émissaire. En outre, les Slovènes ont développé ces attitudes de blâme non seulement dans ses relations extérieures, mais aussi dans ses relations intérieures, entre Slovènes. Ainsi, par exemple, d'un côté, le communisme a identifié les causes des problèmes de la nation dans la religion, alors que, de l'autre, la religion les a vues dans le communisme. Il est vraisemblable qu'une petite nation soit confrontée à de nombreux obstacles et frustrations sur la voie de l'obtention et, plus tard, de la préservation de son indépendance, car ces possibilités sont conditionnées par divers facteurs externes et internes; par conséquent, les gens peuvent facilement faire face à de nombreux obstacles: ceux du niveau de vie, de la sécurité des plus pauvres, de la corruption généralisée et du manque de succès pour l'éradiquer. L'absence de reconnaissance des opportunités, prometteuses de succès éventuels, a contribué à ramener l'explication à une seule cause, et, par conséquent, à l'apparition du bouc émissaire. Burte, ${ }^{17}$ qui a étudié la question des sociétés post-autoritaires, soutient que dans de telles sociétés, après une longue période de régime dictatorial, des droits socio-psychologiques brimés surgissent spontanément au moment où la domination cesse. Cela contribue de manière significative à exacerber la virulence du processus du bouc émissaire.

Les personnes ou les communautés qui ont connu l'expérience du bouc émissaire portent une faible estime de soi et sont divisées pour trouver une solution qu'ils appréhendent. La peur peut même s'intensifier si bien que la communauté devient agressive envers ceux qui s'efforcent de trouver une solution. Si cela persiste assez longtemps, les gens, paradoxalement, s'attachent à de tels ressentiments. Bien qu'ils se trouvent dans une situation difficile, ils ne veulent pas s'en sortir, sous de fallacieux prétextes: « Nous n’y pouvons rien ». Ou: « Ça

17 J. M. Burte, Political Violence and the Authoritarian State in Peru: Silencing Political Society, New York 2010, Palgrave, p. 86. 
n'a aucun sens d'essayer quelque initiative nouvelle». «Bruxelles s'impose, nous n'avons rien à redire ". Au lieu de relever ses manches, ils deviennent encore plus passifs: «Peut-être quelqu'un va-t-il faire quelque chose; moi, je reste en retrait.» Avec de tels individus, le pessimisme, le grognement et les autres formes d'agressivité passive se propagent. Le chemin pour s'en sortir n'est pas simple: chercher des ressources et des signes d'espoir au lieu d'apitoiement sur son sort; s'efforcer de trouver des voies d'avenir, plutôt que de les laisser passivement aux autres; se guérir de ses attitudes de victime et redécouvrir un sentiment de renoncement communautaire.

\section{Conclusion}

Le manque de prise de conscience de ses propres imperfections et la projection de ses faiblesses sur les autres est habituellement la cause majeure pour faire un bouc émissaire. Ainsi, même des personnes dotées de grande compétence et de capacité d'action, et souvent dans des positions d'exercer du leadership, peuvent être facilement victimes de ce mécanisme si elles ne sengagent pas dans la poursuite d'une croissance personnelle.

Cependant, lorsque les gens prennent conscience de leurs zones dombres et de leurs faiblesses, cela peut également créer des tensions internes et un besoin de les évacuer positivement. Les gens doivent être renseignés et guidés pour faire face, d'une manière appropriée, aux tensions, aux frustrations, à la peur, à l'insécurité, à la colère et à des sentiments semblables; sinon, l'une des formes les plus immédiates d'agression mortifère se tourne contre des personnes impuissantes ou innocentes.

Le sens tragique de la vie et de l'apitoiement sur son sort peut empêcher les gens de faire les efforts nécessaires pour changer. Cela fortifie davantage la perception de soi comme victimes, et alimente les attitudes égoïstes.

\section{Bibliography}

Burte J.M., Political Violence and the Authoritarian State in Peru: Silencing Political Society, New York 2010, Palgrave.

Campbell J., Reflections on the Art of Living, San Anselmo, CA 2011, Campbell.

Douglas T., Scapegoats - Transferring Blame, London 1995, Routledge. 
Halbertal M., On Sacrifice, Princeton 2012, Princeton University Press.

Kettle D., Engaging with Tragic Spirituality and Victim Sensibility: on the Cultural Setting of Mission in the West Today, in: Mission Studies 21, p. 287-312.

Mauriac F., Child Martyrs (Second Thoughts), New York 1961, Publisher unknown.

Liersch M.J., I am a Better Driver than You Think: Examining Self-Enhancement for Driving Ability, "Journal of Applied Social Psychology" (2014) 43 (8), p. 1648-1659.

Raven R., The Dorr War - Treason, Rebellion and the Fight for Reform in Rhode Island, Charleston 2010, Narrative Press.

Storr A., Human Aggression, New York 1968, Free Press.

Zeldin T., An Intimate History of Humanity, New York 1995, Harper Perennial.

Zitek E., Victim Entitlement to Behave Selfishly, "Journal of Personality and Social Psychology", February 2010, 98 (2), p. 45-255. 